\title{
Flowthrough Pretreatment of Softwood under Water-only and
}

\author{
Alkali Conditions \\ Libing Zhang ${ }^{1}$, Zhangyang Xu${ }^{1}$, John R. Cort ${ }^{2}$, Tapani Vuorinen ${ }^{3}$, and Bin Yang ${ }^{1,2,3}$ *
}

1. Bioproducts, Sciences, and Engineering Laboratory, Department of Biological Systems

Engineering, Washington State University, Richland, Washington 99354, United States.

2. Earth and Biological Sciences Directorate, Pacific Northwest National Laboratory, Richland, Washington 99354, United States.

3. Department of Bioproducts and Biosystems, Aalto University, P.O. Box 16300, Aalto 00076, Finland.

* Corresponding author: Prof. Bin Yang

E-mail: bin.yang@wsu.edu; Phone: 509-372-7640, Fax: 509-372-7690.

Bioproduct Sciences and Engineering Laboratory, Department of Biological Systems

Engineering, Washington State University, Richland, Washington 99354, United States. 


\section{Supplementary Materials}

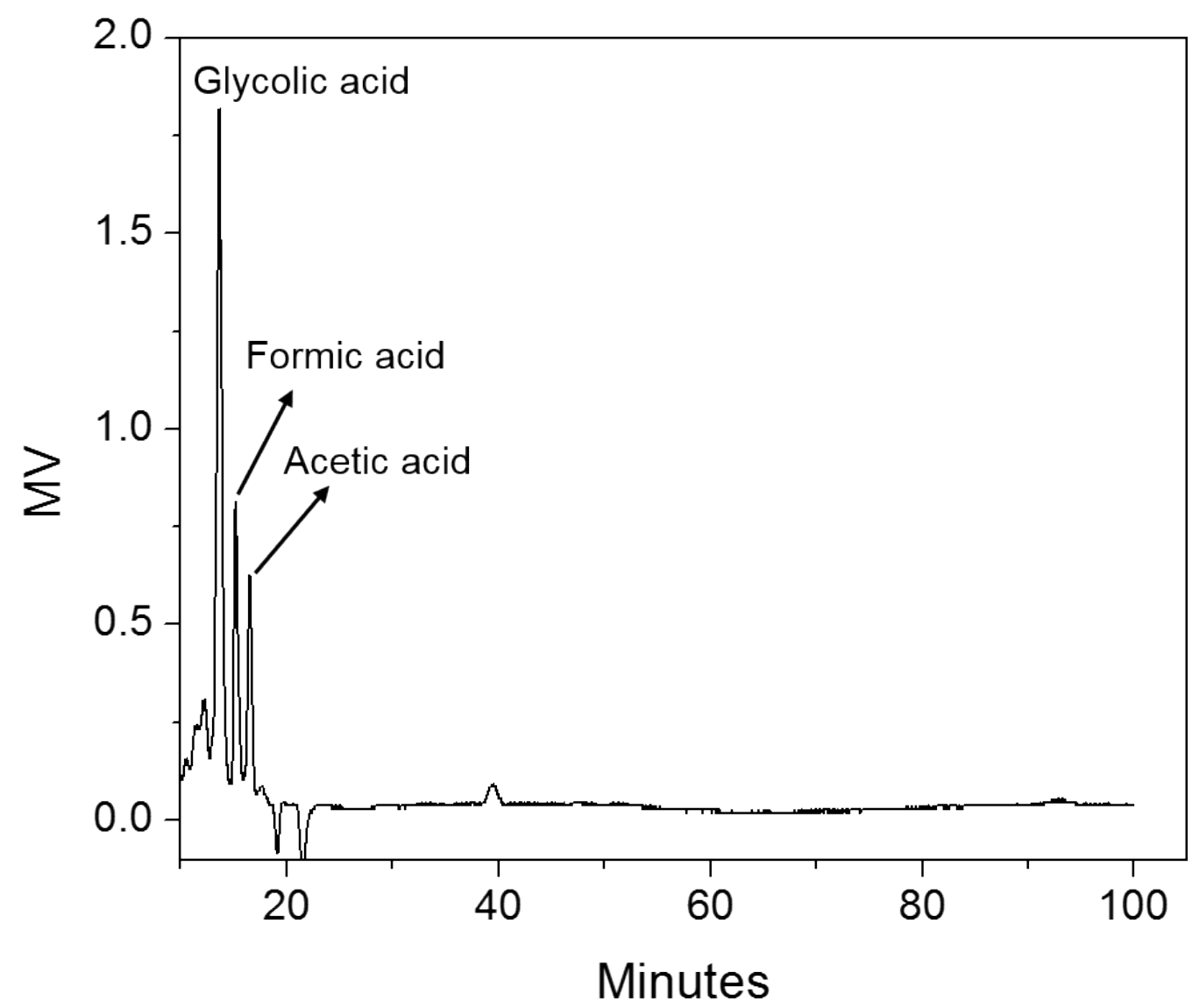

Figure S1. HPLC analysis of degradation products in pretreatment hydrolysates at $\mathrm{pH}$ of 12.0 and pretreatment severity of 6.1 . 


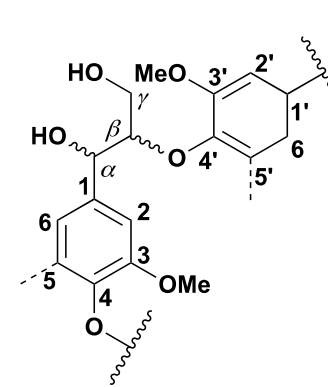

A: B Aryl ether (ß-0-4')

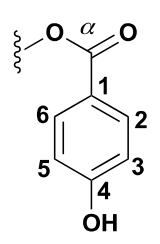

E: p-hydroxybenzoate

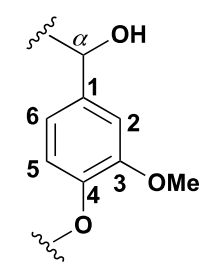

G: guaiacyl units

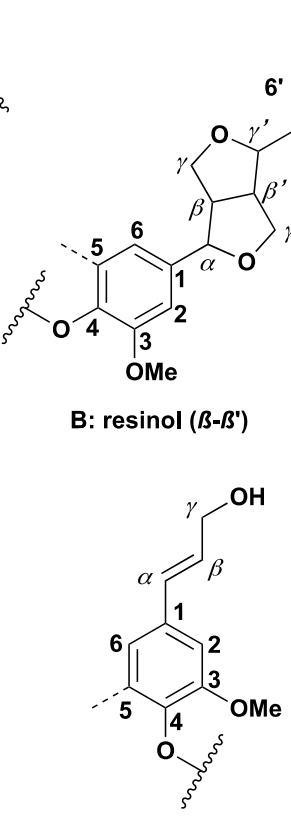

F: p-hydroxycinnamyl alcohol end-groups

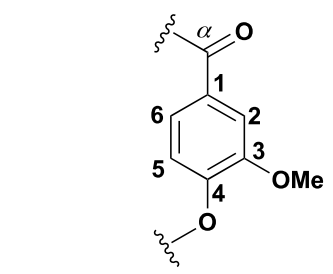

G': oxidized $\left(\mathrm{C}_{\alpha=0}=\mathrm{)}\right.$ guaiacyl units

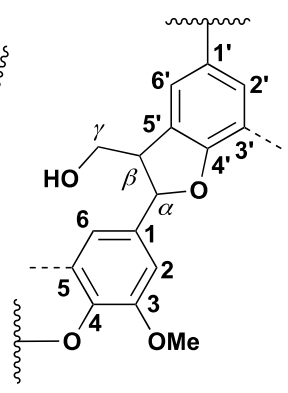

C: phenylcoumaran (ß-5')

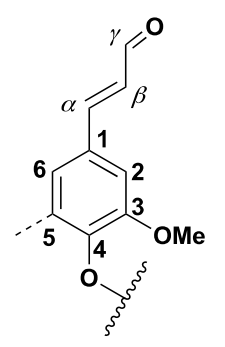

$\mathrm{K}$ : cinnamyl aldehyde end-groups

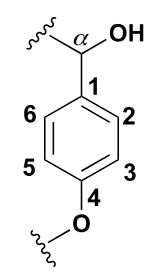

H: p-hydroxyphenyl units

Figure S2. Main detected lignin linkages. 
Table S1. Main lignin 2D ${ }^{1} \mathrm{H}-{ }^{13} \mathrm{C}$ cross-peak assignments in the HSQC spectra.

\begin{tabular}{|c|c|c|c|}
\hline Label & $\delta_{H}(\mathbf{p p m})$ & $\delta_{\mathrm{C}}(\mathbf{p p m})$ & Assignment \\
\hline$-\mathrm{OCH}_{3}$ & 3.73 & 56.0 & $\mathrm{C}-\mathrm{H}$ in methoxyls \\
\hline $\mathrm{A}_{\alpha}$ & 4.71 & 71.3 & $\mathrm{C}_{\alpha}-\mathrm{H}_{\alpha}$ in $\beta$-O-4' substructures (A) \\
\hline $\mathrm{A}_{\gamma}$ & $3.39-3.42$ & $60.3-60.5$ & $\mathrm{C}_{\gamma}-\mathrm{H}_{\gamma}$ in $\beta$-O-4' substructures (A) \\
\hline $\mathrm{A}_{\beta(\mathrm{G} / \mathrm{H})}$ & 4.29 & 84.0 & $\mathrm{C}_{\beta}-\mathrm{H}_{\beta}$ in $\beta-\mathrm{O}-4^{\prime}$ substructures linked to $\mathrm{G}$ and $\mathrm{H}$ units (A) \\
\hline $\mathrm{A}_{\beta(\mathrm{S})}$ & 4.22 & 84.8 & $\mathrm{C}_{\beta}-\mathrm{H}_{\beta}$ in $\beta$-O-4' substructures linked to $\mathrm{S}$ units (A) \\
\hline $\mathrm{B}_{\alpha}$ & 4.65 & 85.4 & $\mathrm{C}_{\alpha}-\mathrm{H}_{\alpha}$ in resinol $\left(\beta-\beta^{\prime}\right)$ substructures $(\mathrm{B})$ \\
\hline $\mathrm{B}_{\beta}$ & 3.05 & 54.0 & $\mathrm{C}_{\beta}-\mathrm{H}_{\beta}$ in resinol $\left(\beta-\beta^{\prime}\right)$ substructures $(\mathrm{B})$ \\
\hline $\mathrm{B}_{\gamma}$ & 3.80 and 4.16 & 71.6 & $\mathrm{C}_{\gamma}-\mathrm{H}_{\gamma}$ in resinol $\left(\beta-\beta^{\prime}\right)$ substructures $(\mathrm{B})$ \\
\hline $\mathrm{C}_{\alpha}$ & 5.43 & 87.4 & $\mathrm{C}_{\alpha}-\mathrm{H}_{\alpha}$ in phenylcoumaran substructures $(\mathrm{C})$ \\
\hline $\mathrm{C}_{\beta}$ & 3.43 & 53.6 & $\mathrm{C}_{\beta}-\mathrm{H}_{\beta}$ in phenylcoumaran substructures $(\mathrm{C})$ \\
\hline $\mathrm{C}_{\gamma}$ & 3.68 & 63.1 & $\mathrm{C}_{\gamma}-\mathrm{H}_{\gamma}$ in phenylcoumaran substructures $(\mathrm{C})$ \\
\hline $\mathrm{E}_{2 / 6}$ & 7.67 & 131.8 & $\mathrm{C}_{2,6}-\mathrm{H}_{2,6}$ in $p$-hydroxybenzoate substructures $(\mathrm{E})$ \\
\hline $\mathrm{F}_{\gamma}$ & 4.09 & 61.9 & $\mathrm{C}_{\gamma}-\mathrm{H}_{\gamma}$ in $p$-hydroxycinnamyl alcohol end-groups $(\mathrm{F})$ \\
\hline $\mathrm{G}_{2}$ & 6.97 & 111.5 & $\mathrm{C}_{2}-\mathrm{H}_{2}$ in guaiacyl units $(\mathrm{G})$ \\
\hline $\mathrm{G}_{5}$ & 6.74 & 115.5 & $\mathrm{C}_{5}-\mathrm{H}_{5}$ in guaiacyl units $(\mathrm{G})$ \\
\hline $\mathrm{G}_{6}$ & 6.76 & 119.1 & $\mathrm{C}_{6}-\mathrm{H}_{6}$ in guaiacyl units $(\mathrm{G})$ \\
\hline $\mathrm{G}_{2}^{\prime}$ & 7.38 & 111.0 & $\mathrm{C}_{2}-\mathrm{H}_{2}$ in oxidized $\left(\mathrm{C}_{\alpha}=\mathrm{O}\right)$ guaiacyl units $\left(\mathrm{G}^{\prime}\right)$ \\
\hline $\mathrm{G}_{6}^{\prime}$ & 7.5 & 123.4 & $\mathrm{C}_{6}-\mathrm{H}_{6}$ in oxidized $\left(\mathrm{C}_{\alpha}=\mathrm{O}\right)$ guaiacyl units $\left(\mathrm{G}^{\prime}\right)$ \\
\hline $\mathrm{H}_{2 / 6}$ & 7.18 & 128.3 & $\mathrm{C}_{2,6}-\mathrm{H}_{2,6}$ in $p$-hydroxyphenyl units $(\mathrm{H})$ \\
\hline $\mathrm{K}_{\beta}$ & 6.77 & 126.7 & $\mathrm{C}_{\beta}-\mathrm{H}_{\beta}$ in cinnamyl aldehyde end-groups $(\mathrm{K})$ \\
\hline $\mathrm{X}_{2}$ & 3.02 & 73.0 & $\mathrm{C}_{2}-\mathrm{H}_{2}$ in $\beta$-D-xylopyranoside \\
\hline $\mathrm{X}_{3}$ & 3.23 & 74.4 & $\mathrm{C}_{3}-\mathrm{H}_{3}$ in $\beta$-D-xylopyranoside \\
\hline $\mathrm{X}_{4}$ & 3.49 & 75.8 & $\mathrm{C}_{4}-\mathrm{H}_{4}$ in $\beta$-D-xylopyranoside \\
\hline $\mathrm{X}_{5}$ & 3.16 & 63.5 & $\mathrm{C}_{5}-\mathrm{H}_{5}$ in $\beta$-D-xylopyranoside \\
\hline $\mathrm{X} 2_{2}$ & 4.49 & 73.7 & $\mathrm{C}_{2}-\mathrm{H}_{2}$ in 2-O-acetyl- $\beta$-D-xylopyranoside \\
\hline $\mathrm{X} 3_{3}$ & 4.78 & 75.3 & $\mathrm{C}_{3}-\mathrm{H}_{3}$ in 3-O-acetyl- $\beta$-D-xylopyranoside \\
\hline
\end{tabular}

